\title{
Different types of "dissociation" have different psychological mechanisms
}

\author{
Richard J. Brown, Ph.D, Clin.Psy.D
}

\section{Academic Division of Clinical Psychology, University of Manchester}

Address for correspondence: Dr Richard J. Brown, Academic Division of Clinical Psychology, University of Manchester, Rawnsley Building, Manchester Royal Infirmary, Oxford Road, Manchester, M13 9WL, UK. E-mail: richard.james.brown@manchester.ac.uk

Published in Journal of Trauma and Dissociation, 7, 7-28, 2006 


\begin{abstract}
The term "dissociation" has been used to describe a wide range of psychological and psychiatric phenomena. The popular conception of dissociation describes it as a unitary phenomenon, with only quantitative differences in severity between the various dissociative conditions. More recently, it has been argued that the available evidence is more consistent with a model that identifies at least two distinct categories of dissociative phenomena "detachment" and "compartmentalization" - that have different definitions, mechanisms and treatment implications (Holmes, Brown, Mansell, Fearon, Hunter, Frasquilho \& Oakley 2005). This paper presents evidence for this bipartite model of dissociation, followed by definitions and descriptions of detachment and compartmentalization. Possible psychological mechanisms underlying these phenomena are then discussed, with particular emphasis on the nature of compartmentalization in conversion disorder, hypnosis, dissociative amnesia and dissociative identity disorder.
\end{abstract}




\section{INTRODUCTION}

When the term "dissociation" was originally popularised in the $19^{\text {th }}$ century, it was used to refer to a putative mental mechanism underlying a relatively circumscribed set of clinical phenomena (Van der Hart \& Dorahy, in press). Since the renaissance of the concept in the 1970s, however, and the growth of contemporary theories (e.g., Hilgard, 1977) concerning the nature of this mental mechanism, the number of phenomena thought to be attributable to dissociation has expanded considerably. As a result, the dissociation label is now applied to an extraordinary range of psychological symptoms, states and processes (see figure 1; Cardeña, 1994).

\section{INSERT FIGURE 1 ABOUT HERE}

On the face of it, this expansion of the dissociative domain (Cardeña, 1994) appears to be justified by the widespread view that these different phenomena described above are produced by a common psychological mechanism (i.e. dissociation), characterised by a breakdown in mental integration (e.g., Bernstein \& Putnam, 1986; Dell, in press). According to this "unitary" model, these phenomena are all qualitatively similar, with the differences between them being accounted for by the "amount" of dissociation involved in each case. This idea is embodied in the concept of the so-called dissociative continuum (figure 2) and forms the basis for the Dissociative Experiences Scale (DES; Bernstein \& Putnam, 1986), which is commonly used to estimate individual differences in "trait" dissociation. The model is also apparent in the DSMIV definition of dissociation, which identifies it as “... a disruption in the usually integrated functions of consciousness, memory, identity or perception of the environment" (p. 477; APA, 1994). The unitary model is able to account for a large body of research findings demonstrating that the DES scores of different clinical groups vary as predicted, with the most disabling conditions (such as DID) being 
associated with the highest DES scores (see Van Ijzendoorn \& Schuengel, 1996). In addition, the model provides a parsimonious and accessible account of the available clinical data and is therefore intuitively appealing to both clinicians and patients.

\section{INSERT FIGURE 2 ABOUT HERE}

Despite the appeal of the unitary model, it is not without its critics (see e.g., Cardeña, 1994; Frankel, 1990, 1994; Van der Hart, Nijenhuis, Steele \& Brown 2004). According to Frankel $(1990,1994)$, for example, the dissociation concept has been over-extended to encompass almost any kind of symptom involving an alteration in consciousness or a loss of mental or behavioural control. Similarly, Holmes et al (2005) have argued that the unitary model is based on a definition of dissociation that is too broad and which obscures fundamental differences between the various phenomena that it encompasses. If valid, this argument has far-reaching implications. Empirically, it implies that researchers need to move beyond simply recruiting generic groups of patients with "dissociative disorders" and instead focus on the specific symptoms or symptom clusters in question. Clinically, it indicates that different types of treatment may be required for different dissociative problems, and that the "onesize-fits-all" approach implied by the unitary model is invalid.

Numerous previous theorists have attempted to address the confusion caused by the unitary model by identifying different and separate "types" of dissociation (Cardeña, 1994; Allen, 2001; Putnam, 1997; Brown, 2002a; Van der Kolk \& Fisler, 1995). Holmes et al (2005) provide a summary position based on these previous theories, arguing that the available evidence is consistent with a model that distinguishes between two qualitatively different kinds of phenomena - "detachment" and "compartmentalization" (following Allen, 2001) - each with distinct definitions, mechanisms and treatment implications. In the first half of this paper, I will describe 
the Holmes et al (2005) model, providing definitions and descriptions of detachment and compartmentalization, as well as evidence supporting a distinction between these phenomena. In the second half, I will elaborate on the Holmes et al (2005) model by relating it to a recent account of the cognitive mechanisms underlying compartmentalization (Brown, 2002a, 2004; Brown \& Oakley, 2004).

\section{EVIDENCE FOR TWO TYPES OF PATHOLOGICAL “DISSOCIATION"}

Although research investigating total scores on the DES seems to support a unitary model of dissociation, factor analytic studies of the measure are more consistent with a multifactorial account. Numerous studies have found that the DES has a complex factor structure with at least three underlying factors (e.g., Carlson, Putnam, Ross et al., 1991; Ross, Ellason \& Anderson 1995; Frischolz, Braun, Sachs, et al., 1991; Ross, Joshi \& Currie 1991). Almost invariably, such studies have identified separate factors for absorption, depersonalization-derealization, and amnesia related items, suggesting that these three forms of "dissociation" do not belong to the same category of phenomena. One possible explanation for this pattern of findings is that absorption, depersonalization-derealization, and amnesia occur at different rates in the population, producing a misleading multi-factorial solution when the DES is factor-analyzed (Bernstein, Ellason, Ross \& Vanderlinden 2001). This would be consistent with the widespread view that absorption is a common and non-pathological phenomenon experienced by most people to varying degrees, unlike "pathological" forms of dissociation such as amnesia, depersonalization-derealization and identity alteration (e.g., Waller, Putnam \& Carlson 1996). While this could explain why a separate absorption factor was identified in the Bernstein et al (2001) study, it is less clear how it could account for the identification of separate amnesia and depersonalization- 
derealization factors, particularly as there was no evidence that these two had different base-rates in the general population sample that was assessed.

According to Holmes et al (2005), the statistical separation of amnesia and depersonalization-derealization items on the DES reflects the fact that these phenomena belong to two qualitatively different categories of experience compartmentalization and detachment respectively - that can be distinguished both empirically and conceptually. Consistent with this, Holmes et al (2005) point to a number of other studies suggesting that depersonalization and derealization can be separated from amnesia and other "dissociative" phenomena, such as somatoform symptoms ${ }^{2}$ and comparable experiences produced using hypnotic suggestion. Research on depersonalization disorder, for example, demonstrates that medically unexplained (i.e. somatoform) symptoms are relatively rare in this group (Baker et al., 2003). Similarly, patients with depersonalization disorder fall in the average range on a sub-scale of the DES made up of amnesia-related items, despite having elevated scores on a depersonalization-derealization sub-scale (Simeon et al., 2003). Furthermore, depersonalization and derealization are relatively uncommon in patients with medically unexplained symptoms (Brown, Schrag \& Trimble 2005). In contrast, patients with amnesia and dissociative disorders such as DID often report somatoform symptoms (Saxe et al., 1994; Nijenhuis, 2004). Patients with somatoform symptoms often yield low scores on the DES, however, because the scale has relatively few items pertaining to amnesia and other examples of compartmentalization (Brown, 2005). Indeed, the somatoform dissociation questionnaire (SDQ-20) was developed to rectify this omission in the DES (Nijenhuis 2004).

Probably the strongest support for the distinction between depersonalizationderealization and other types of "dissociative" phenomena comes from research 
addressing their mechanisms. This will be considered in some detail below, following definitions and descriptions of detachment and compartmentalization as described in the Holmes et al (2005) model.

\section{DETACHMENT}

\section{$\underline{\text { Definition and description of detachment }}$}

Holmes et al (2005) define detachment as an altered state of consciousness characterized by a sense of separation (or 'detachment') from aspects of everyday experience (see also Cardeña, 1994; Allen, 2001). The sense of detachment may relate to the individual's emotional experience (as in emotional numbing), their sense of self (as in some depersonalization phenomena), their body (as in out-of-body phenomena), or the world around them (as in derealization; see table 1). The phenomena may occur in isolation although they commonly co-occur. In each case, the individual's sense of reality testing during the detachment experience is preserved. Phenomenological descriptions of detachment include an absence or alteration of emotional experience, feelings of being "spaced out", "disconnected", "unreal" or "in a dream", a sense of being an outside observer of one's body, and perceptions of the external world as flat, lifeless and "strange" (Noyes \& Kletti, 1977; Steinberg, 1994; Butler, Duran, Jasiukaitis, Koopman \& Spiegel 1996; Allen, Console \& Lewis 1999; Sierra \& Berrios, 2001; Baker et al., 2003). In some cases, detached states are associated with memory disturbances and amnesia (Allen et al., 1999). On the face of it, detachmentrelated memory dysfunction can be difficult to distinguish from amnesia as a compartmentalization phenomenon. According to the Holmes et al (2005) model, however, the mechanisms responsible for detachment-related memory loss are different to those operating in compartmentalization (see below). 
Detachment phenomena may manifest as a disorder in their own right, as in depersonalization disorder, or in the context of another condition, such as an anxiety or affective disorder (Hunter, Sierra \& David 2004). Detachment is also commonly experienced during, or immediately after, traumatic events, a phenomenon known as "peri-traumatic dissociation", which is a defining feature of acute stress disorder (ASD) in DSM-IV ${ }^{3}$. In addition, many individuals report mild and transient detachment experiences during periods of fatigue, intoxication or stress. As such, detachment phenomena can be arranged on a continuum of increasing distress and disability, ranging from mild and non-pathological experiences of detachment to extremely disabling symptoms, such as those seen in depersonalization disorder.

In the original Holmes et al (2005) model, all depersonalization phenomena were regarded as examples of detachment. In the formulation of the model that is outlined here, however, "made" actions, which are routinely identified as examples of depersonalization, are regarded as cases of compartmentalization (see below).

\section{$\underline{\text { Mechanisms of detachment }}$}

Holmes et al (2005) follow Sierra and Berrios (1998) in assuming that detached states result from a hard-wired biological defence mechanism evolved to minimize the potentially debilitating effects of extreme affect in threatening situations. By this view, detachment arises when an increase in anxiety causes the medial prefrontal cortex to inhibit emotional processing by the limbic system, thereby reducing sympathetic output (Sierra \& Berrios, 1998). The result is a state devoid of emotional experience that facilitates adaptive behavior in the face of threat. Although this detached state is adaptive in the short term, it may be highly aversive and debilitating if it persists over time, as in depersonalization disorder. Hunter, Phillips, Chalder, Sierra \& David (2003) suggest that chronicity may develop when the individual 
misinterprets the state of detachment itself as a threat (e.g., of impending mental breakdown), perpetuating anxiety and emotional inhibition.

There is a growing body of evidence in favour of this account of the mechanisms of detachment. It is well documented, for example, that depersonalization and derealization are commonly associated with anxiety, both normal (e.g., Sterlini \& Bryant, 2002) and pathological (e.g., Cassano et al., 1989; Simeon, Gross, Guralnik, Stein, Schmeidler \& Hollander 1997; Marshall, Schneier, Lin, Simpson, Vermes \& Leibowitz 2000). Compared to normal and anxious control participants, depersonalization disorder patients also show significantly reduced skin conductance amplitudes and increased skin conductance latencies (both measures of emotional reactivity) to unpleasant stimuli, but not to neutral, unpleasant or non-specific stimuli (Sierra et al., 2002). Similarly, compared to anxious and normal controls, depersonalization disorder patients show reduced neural responses in brain regions typically activated by emotional stimuli (insula and occipto-temporal cortex) and increased neural responses in regions associated with emotional regulation (right ventral prefrontal cortex) when exposed to aversive pictures (Phillips et al., 2001). Other evidence provides indirect support for the idea that detachment is associated with a hard-wired neurophysiological profile, including the stability of depersonalization disorder semiology over time (Sierra \& Berrios, 2001) and the occurrence of detachment phenomena in neurological conditions and drug states (Lambert, Sierra, Phillips \& David 2002).

\section{COMPARTMENTALIZATION}

\section{Definition and description of compartmentalization}

Following Cardeña (1994), Holmes et al (2005) provide the following definition of compartmentalization phenomena: (i) the phenomenon involves a deficit in the ability 
to deliberately control processes or actions that would normally be amenable to such control; (ii) the deficit cannot be overcome by an act of will; (iii) the deficit is reversible, at least in principle; and (iv) it can be shown that the apparently disrupted functions are operating normally and continue to influence cognition, emotion and action. This definition encompasses dissociative amnesia, fugue, DID and the various physical symptoms characteristic of the conversion disorders and some somatoform disorders (e.g., somatization disorder; for other examples of "somatoform dissociation" see Nijenhuis, 2004). Similar phenomena (i.e. amnesia, anaesthesia, pseudohallucinations, motor disturbances etc.) that can be produced using hypnotic suggestion are also included in this category (see Oakley, 1999; Brown, 2004). In addition, unlike the original formulation of the Holmes et al (2005) model, the current account of compartmentalization also encompasses actions that the individual does not feel they are controlling (so-called "made" actions; Dell, 2004), which are typically regarded as examples of depersonalization (see e.g., Steinberg, 1994).

Compartmentalization phenomena can also be regarded as occupying their own continuum of distress and disability, ranging from non-pathological experiences produced using hypnotic suggestion, through milder pathological states such as transient amnesias and conversion disorders, to chronic and extremely disabling conditions like somatization disorder and DID. In each case, the apparently disrupted functions are said to be "compartmentalized".

Rather than providing a detailed account of the psychological mechanisms underlying compartmentalization, Holmes et al (2005) describe laboratory examples that illustrate this phenomenon and distinguish it from detachment. Probably the most compelling empirical demonstration of pathological compartmentalization is an innovative study by Kuyk, Spinhoven and Van Dyck (1999). Kuyk et al (1999) 
compared a group of patients with amnesia following generalized non-epileptic seizures (NES; a form of compartmentalization according to the current scheme) and a group with amnesia following generalized epileptic seizures (ES). Participants in both groups were hypnotized some time after a seizure and given suggestions for the recovery of memories concerning events occurring during the ictus. All information recovered using this procedure was corroborated independently. On this basis, Kuyk et al (1999) found that $85 \%$ of their NES patients recalled information about the seizure for which they were previously amnesic, compared to $0 \%$ of patients in the ES group. The findings of this study clearly demonstrate that the NES patients had encoded information about events occurring during their seizure, but the compartmentalization of this information within the cognitive system had rendered it unavailable for deliberate retrieval. Hypnotic suggestion had later been able to overturn this retrieval failure, allowing recall to take place. The ES patients, in contrast, did not recover ictal information following hypnosis presumably because a generalized brain dysfunction had prevented material from being encoded during the seizure (Brown, 2002b). Following Allen et al., (1999), we assume that amnesia associated with a period of profound detachment also reflects an encoding failure, thereby distinguishing it from the inability to retrieve stored information in amnesia associated with compartmentalization. At present, this remains a conceptual assumption that requires empirical validation.

Other published demonstrations of compartmentalization can be found in single case studies of so-called implicit perception (Kihlstrom, 1992) in conversion disorder patients. Bryant and McConkey (1989), for example, tested a patient (DB) with unilateral conversion blindness using a forced-choice visual decision paradigm. In this, DB was presented with three visual stimuli (triangles) to his affected eye and he 
was asked to generate a response indicating which of the three stimuli was oriented differently to the other two. Using this procedure, Bryant and McConkey (1989) showed that DB responded correctly on $74 \%$ of the trials, an above-chance response rate indicating that his performance was influenced by the visual information available, despite the fact that he continued to report a lack of visual experience in his affected eye. This is clearly consistent with the definition of compartmentalization outlined above. DB's response rate also improved following feedback suggesting that his performance was affected by the visual information and improved further still when he was given instructions designed to increase his motivation. These latter findings indicate that the nature of the compartmentalization associated with DB's blindness was relatively fluid and subject to modification through top-down influences. Other single-case studies of implicit perception associated with compartmentalization are described in Kihlstrom (1992).

Although the study reported by Kuyk et al (1999) and single case studies such as that of Bryant and McConkey (1989) provide compelling examples of compartmentalization, they do not provide a detailed explanation of the mechanisms underlying these phenomena, which is largely lacking from the Holmes et al (2005) model. In a bid to address this issue, the second half of this paper extends the Holmes et al (2005) model by relating it to the integrative cognitive model described by Brown (2002a, 2004; Brown \& Oakley, 2004; also Oakley, 1999a, b), which has been used recently to account for a range of compartmentalization phenomena.

\section{$\underline{\text { Mechanisms of compartmentalization }}$}

The integrative cognitive model was originally developed as an account of the mechanisms underlying certain somatoform symptoms as well as comparable experiences produced using hypnotic suggestion, both of which are examples of 
compartmentalization according to Holmes et al (2005). The integrative model is based on the assumption that these phenomena result from subtle disturbances in the processes underlying consciousness and mental control. To this end, the model provides a detailed account of the cognitive structures and processes associated with normal consciousness and control, which is then applied to atypical cases such as compartmentalization phenomena.

\section{Consciousness and cognitive control}

The basic cognitive architecture according to this approach is presented in figure 3 .

\section{INSERT FIGURE 3 ABOUT HERE}

In line with much cognitive theorising, the model assumes that the contents of consciousness represent a working interpretation of the environment that is generated for the control of cognition and action. In this architecture, the contents of consciousness are generated at a relatively late stage in the processing chain, following extensive pre-attentive analysis of incoming information (Velmans, 2000). In the first instance, the receipt of sensory information triggers simple perceptual analyses that represent its basic features (Kosslyn, 1996). The resulting representations are then encoded in memory, triggering a parallel spread of activation through related representations within memory (Logan, 1988). This spread of activation in associative memory acts as an interpretive process (Sloman, 1996) that produces a number of possible "hypotheses" about the input based on previous experience (Marcel, 1983). These hypotheses are repeatedly sampled by a primary attentional system (PAS), which selects one of the hypotheses as the most appropriate account of the current situation. The PAS then integrates the chosen hypothesis with relevant sensory information, producing multi-modal units or primary representations. These primary representations correspond to the basic contents of 
consciousness and provide a working model of the world that can be used to guide action. Primary representations serve as input to a hierarchical network of behavioral programs or "schemata" (cf. Hilgard, 1977; Norman \& Shallice, 1986) that describe the processing operations required for the execution of specific acts. At the top of this hierarchy are high-level programs corresponding to general situations such as "driving a car" or "going to a restaurant". Within each of these high-level programs are simpler schemata corresponding to different acts within that situation, such as "reversing" or "ordering food". Each of these schemata has even simpler sub-programs describing the various elements of the act (e.g., "changing gear" or "reading the menu") and so on. These programs are activated to varying degrees by the current primary representation. When a threshold level of activation is reached, the program is triggered automatically and the associated behavior is executed using the primary representation as a template of the current environment. This behavior will then run until completion unless it is impeded or inhibited by other information in the system. This automatic activation of schemata provides the system with an efficient way of controlling processing in routine situations and is comparable to the contention scheduling mechanism described by Norman and Shallice (1986). Processes controlled by the automatic activation of schemata are regarded as voluntary but unwilled (cf. Jahanshahi \& Frith, 1998). Processing at this level is perceived as effortless and is associated with an intuitive or pre-reflective subjective character labelled primary awareness.

In cases where the network of behavioral programs is unable to produce adaptive behaviour, such as novel situations, a secondary attentional system $\left(\mathrm{SAS}^{4}\right)$ may intervene to bias the activation levels of relevant programs. The SAS operates via general-purpose problem-solving algorithms, the construction of goals (or secondary 
representations) and the analysis and manipulation of primary representations. Primary representations that are subjected to focal-attentive processing (Velmans, 2000) by the SAS are in the foreground of perceptual experience; those that are not currently being processed by the SAS form the perceptual background. Processing controlled by the SAS is willed (cf. Jahanshahi \& Frith, 1998), effortful, deliberate and associated with a subjective character of self-awareness (i.e. an awareness of being awareness) labelled secondary awareness.

This model of the cognitive architecture has important implications for the explanation of somatoform symptoms, hypnotic experiences and other types of compartmentalization phenomena. In particular, the model assumes that sensation and the contents of experience need not match, as the latter are shaped by both sensory and memorial information and may therefore be "over-determined" by memory. Any discrepancies between sensation and experience will not be experienced directly by the individual (although they may be inferred post hoc) as the processes involved in the creation of experience are unavailable to introspection. As a result, the individual may engage in behaviour that is consistent with the interpretation of events that is currently dominating their experience, irrespective of whether that interpretation is correct. The model also assumes that many, if not most, behaviours are governed by the automatic activation of behavioral programs (i.e. the PAS) rather than via deliberate selection and control by the individual (i.e. the SAS). As such, many complex behaviours can be performed with minimal representation in conscious experience (or, at least, self-conscious experience; cf. Hilgard, 1977). In addition, there can be significant discrepancies between automatically controlled behaviours and goals in self-awareness.

\section{Compartmentalization phenomena and incongruous memory retrieval}


According to the current approach, all compartmentalization phenomena are similar in that they arise from disturbances in the memory retrieval processes associated with the construction of consciousness and/or automatic control of action. By this view, the nature of specific compartmentalization phenomena will vary according to the type of information (or "rogue representation") involved in the retrieval process. Phenomena characterised by a distortion in conscious experience, such as unexplained (or suggested) pain, pseudohallucinations, sensory alterations/loss etc., arise from the retrieval of inappropriate (i.e. inconsistent with sense data) perceptual hypotheses from memory during the creation of primary representations by the PAS. The result is a compelling distortion in the perceptual world related to the content of the inappropriately selected memory. Phenomena characterised by a loss of deliberate control over processes that are normally amenable to such control, such as unexplained (or suggested) paralysis, seizures, urinary retention, amnesia etc., result from the automatic selection of inappropriate behavioral programs corresponding to the experience in question. Thus, amnesia may result from a program specifying the inhibition of certain memory content, while a paralysis may arise from a program inhibiting bodily movements. Behavioral programs may be activated either directly or through the creation of a distorted primary representation that is consistent with the program content (e.g., an experience of stiffness in the arm triggering a program inhibiting arm movement).

Broadly speaking, then, compartmentalization phenomena arise when the cognitive system misinterprets information in memory (i.e. rogue representations) as the most appropriate account of, or response to, current circumstances. Rogue representations can be acquired from a number of different sources, both internal (e.g., direct experience of the symptom, imagery/fantasy, verbal auto-suggestion) and external 
(e.g., exposure to symptoms in others, media images, hetero-suggestion; see Brown, 2004; also Johnson \& Raye, 1981). In each case, the symptom is generated at a late stage in the processing chain, either during the construction of primary representations by the PAS or via the automatic activation of behavioral programs by those representations. As these processes operate prior to focal processing by self-conscious systems (i.e. the SAS), the individual experiences a subjectively compelling deficit that is outside their willed control. Importantly, the apparently damaged functions or systems operate normally prior to attentional selection by the PAS: it is only the conscious representation of these systems' output that is disrupted. Moreover, the affected systems can still influence on-going thought and action by their effect on other, non-affected, aspects of processing. In these senses, symptoms generated in this way can be regarded as archetypal examples of compartmentalization.

The misinterpretation underlying compartmentalization is driven by the overactivation of rogue representations in memory; as such, any factor that increases this activation will increase the likelihood of a rogue representation being selected and therefore moderate the occurrence of compartmentalization (see Brown, 2004; Brown \& Oakley, 2004). In some cases, this may be cognitive factors such as symptom checking, catastrophic thinking and illness worry/rumination; in others, motivational factors (e.g., avoidance of alternative memory content) may be central.

At present, the integrative cognitive model has been applied to medically unexplained symptoms (including amnesia and pseudohallucinations) but not other forms of pathological compartmentalization such as "made" actions. On the face of it, the sense of involuntariness that accompanies such actions is akin to that associated with apparently automatic behaviours in the hypnotic context (the so-called "classic suggestion effect"). According to Brown and Oakley (2004), this phenomenon arises 
when an unwilled act (i.e. one governed by the automatic activation of a behavioral program) is misinterpreted as coming from an external source, due to certain information about that act within the cognitive system. In some cases, this information may be the absence of an advance prediction about the sensory consequences of the act (see Blakemore, Wolpert \& Frith 2002; Blakemore, Oakley \& Frith 2003) and/or the activation of a goal specifying that the behavior should be experienced as involuntary (Kirsch \& Lynn, 1997). In others, it may be the activation of a goal that is inconsistent with the act, particularly if the individual is motivated not to experience it as their own due to its "unacceptability".

\section{The working self and compartmentalization in dissociative amnesia and DID}

One of the basic assumptions of the Holmes et al (2005) model is that similar mechanism are operating in all compartmentalization phenomena. How might the integrative cognitive model account for the various symptoms of DID, such as the occurrence of multiple identities and inter-identity amnesia? Although an extensive discussion of this issue is beyond the scope of this article, some theoretical speculations are in order. As with other aspects of the model, the most appropriate approach is to begin by understanding the nature and development of the self under normal circumstances. The self-memory system model of Conway and PleydellPearce (2000; also Conway, 2005) provides a number of important insights into this process that could be used to develop the integrative cognitive model in this respect. According to Conway and Pleydell-Pearce (2000), the self (or working self in their terminology) consists of a set of hierarchically-organized goals in working memory constructed to reduce discrepancies between current and desired states of the system; these discrepancies are associated with negative emotional experiences, which provide the motivating force behind goal development and maintenance. The working 
self has access to an autobiographical knowledge base that represents information about previous events that pertain to system goals. The retrieval of individual autobiographical memories occurs when a stable pattern of activation develops within the autobiographical knowledge base that is linked to current goals in the working self. This may occur through the creation of a deliberate retrieval plan by the working self or via direct activation of the knowledge base by cues from the environment.

Although developed for quite different purposes, the model of Conway and Pleydell-Pearce's (2000) is consistent with many aspects of the integrative cognitive model described above. Thus, the goal hierarchy that constitutes Conway and Pleydell-Pearce's working self would be an important aspect of the control structures that make up the secondary attentional system, whereas the autobiographical knowledge base would be one part of associative memory. By this view, autobiographical memories will be retrieved when their activation patterns in associative memory are sufficient for them to be selected by the PAS during the creation of primary representations. This may occur via the creation of new retrieval programs by the SAS, the activation of old retrieval programs by primary representations, or the direct activation of associative memory by environmental cues.

One important feature of the Conway and Pleydell-Pearce model that is not explicit in the integrative cognitive model is the idea that autobiographical memory retrieval is strongly constrained by the goals of the working self. According to Conway and Pleydell-Pearce (2000), one important goal of the working self is to limit the retrieval of autobiographical memories that may be destabilizing to the system, such as those that are associated with strong affect and/or highlight discrepancies between goals and knowledge. The working self does this by creating retrieval programs that specify inhibition of memory content of this sort, which remains compartmentalized in the 
system. Conway and Pleydell-Pearce suggest that this may be one route to the development of traumatic amnesia in PTSD, an idea that could conceivably be extended to all forms of dissociative memory loss. The account of dissociative amnesia provided by the integrative cognitive model is entirely consistent with this notion if one assumes that the process is similar whether the inhibitory retrieval program (i.e. rogue representation) is established in memory or created on-line by the SAS $^{5}$.

The idea that self goals determine what is available for autobiographical recall could also help account for the occurrence of dissociative amnesia in patients with DID, although a more complex explanation is probably required here. In normal circumstances, the working self will have a high degree of internal consistency, such that the various goals within the structure are mutually compatible. In cases where there is a discrepancy between conflicting goals (e.g., the goal to develop a romantic relationship vs. the goal to avoid rejection at all costs), negative affect (e.g., anxiety) will arise. This affect can be managed by reducing the discrepancy between the conflicting goals, either through adaptive (e.g., adopting a more realistic goal in relation to rejection) or maladaptive means (e.g., avoiding romantic relationships). In chaotic or traumatic environments, however, it may be impossible to reduce discrepancies between basic behavioral goals (e.g., the goal to be close to attachment figures and the goal to avoid physical or emotional pain). One way for the cognitive system to manage the resulting anxiety would be to prevent the simultaneous activation of the conflicting goals. If this were to happen often enough, separate goal hierarchies (or working selves) could develop over time, each comprising the goals and sub-goals that were co-active with the conflicting goal in question. Each goal hierarchy would have access to the autobiographical memories associated with its 
component goals, while memories associated with the conflicting goal would either be inhibited or unavailable due to the lack of relevant links in the knowledge base ${ }^{6}$. Such a fragmented goal hierarchy could account for the gaps in time experienced by many patients with DID, as well as the occurrence of multiple identities with inter-identity amnesia. The characteristic behavioral pattern of each identity would reflect the type of goals that make up the goal-hierarchy in question. It is likely that these will be organised around fundamental behavioral goals or action tendencies such as those described by Nijenhuis, Van der Hart and Steele (2004).

According to this account, the compartmentalization operating in less pathological phenomena such as simple conversion disorders or circumscribed dissociative amnesias involves a separation (or "dissociation") between different levels of processing within the cognitive system (i.e. the results of low level processing are separated from the SAS by the PAS). In DID, the compartmentalization is not only between the PAS and SAS, but also within SAS structures themselves. In many ways, this distinction is similar to that between the different levels of structural dissociation (i.e. primary and secondary vs. tertiary) in the Nijenhuis et al (2004) model.

This account of compartmentalization in DID is clearly both speculative and in need of further development. Nevertheless, it provides some indication of how the symptoms of DID might be understood using cognitive models such as those of Conway and Pleydell-Pearce (2000) and Brown (2004), suggesting that this may be a fruitful avenue for future investigation.

\section{IMPLICATIONS AND CONCLUSIONS}

The model of Holmes et al (2005) has a number of important empirical and clinical implications. In particular, the model emphasises that total scores on the DES may not be the most useful way of describing the "dissociative" tendencies of subject groups 
and that sub-scales of the DES, or more specific measures of detachment and/or compartmentalization, may be more appropriate for research or clinical purposes. Similarly, the model demonstrates the importance of selecting diagnostically pure groups in research studies in this area, rather than heterogeneous groups of "dissociative disorder" patients (or individuals scoring high on the DES in nonclinical studies). Further evidence for the model could, however, be obtained from cluster analytic studies using mixed diagnostic groups and multiple measures of detachment and compartmentalization. In addition, further studies investigating the mechanisms of detachment and compartmentalization, using cognitive, neurophysiological and neuroimaging methods are urgently required.

Clinically, the model indicates that a "one size fits all" approach to treatment is invalid and highlights the importance of developing idiosyncratic formulations of dissociative disorder patients, based on an understanding of the specific psychological mechanisms of the problem in question. Recent evidence suggests that CBT using an adapted anxiety-disorder model is an effective treatment for pathological detachment (Hunter, Baker, Phillips, Sierra \& David 2005). Other forms of treatment may be more appropriate for pathological compartmentalization (for discussion see Holmes et al., 2005). Finally, the model questions the unqualified use of the term "dissociation" and emphasises the need to be much more specific about the kinds of phenomena that are being referred to when we use the label. 


\section{FOOTNOTES}

1. Or, strictly speaking, desagrégation, which was subsequently translated into its English form.

2. This paper distinguishes between "symptoms" and "experiences". Both of these may be regarded as consciously identifiable subjective events; the assumption here is that only the former is associated with some kind of pathological process. The term "phenomena" is used as a collective label for both symptoms and experiences.

3. Amnesia is also identified as a symptom of peri-traumatic dissociation in ASD. In the Holmes et al (2005) model, amnesia is a non-specific symptom that can be associated with either detachment or compartmentalization (see below).

4. The SAS is comparable to the supervisory attentional system in the Norman and Shallice (1986) model.

5. The on-line generation of inhibitory programs by the SAS may play an important role in hypnotic and post-hypnotic amnesias (see Brown \& Oakley, 2004).

6. The degree to which different working selves would have access to memories related to other selves would presumably also reflect the degree to which the goal hierarchies were in conflict. 


\section{REFERENCES}

Allen, J. G. (2001). Traumatic relationships and serious mental disorders. New York, NY: John Wiley and Sons.

Allen, J. G., Console, D. A., \& Lewis, L. (1999). Dissociative detachment and memory impairment: Reversible amnesia or encoding failure. Comprehensive Psychiatry, 40, 160-171.

American Psychiatric Association. (1994). Diagnostic and Statistical Manual of Mental Disorders (4th ed.). Washington, DC: Author.

Baker, D., Hunter, E., Lawrence, E., Medford, N., Patel, M., Senior, C., et al. (2003). Depersonalisation disorder: Clinical features of 204 cases. British Journal of Psychiatry, 182, 428-433.

Bernstein, E., \& Putnam, F. W. (1986). Development, reliability and validity of a dissociation scale. Journal of Nervous and Mental Disease, 174, 727-735.

Blakemore, S-J., Wolpert, D.M. \& Frith, C. D. (2002). Abnormalities in the awareness of action. Trends in Cognitive Sciences, 6, 237-242.

Blakemore, S-J., Oakley, D. A. \& Frith, C. D. (2003). Delusions of alien control in the normal brain. Neuropsychologia, 41, 1058-67.

Brown, R. J. (2002a). The cognitive psychology of dissociative states. Cognitive Neuropsychiatry, 7, 221-235.

Brown, R. J. (2002b). Epilepsy, dissociation and nonepileptic seizures. In M. R. Trimble, \& B. Schmitz (Eds.), The neuropsychiatry of epilepsy (pp. 189-209). Cambridge, UK: Cambridge University Press.

Brown, R. J. (2004). The psychological mechanisms of medically unexplained symptoms: An integrative conceptual model. Psychological Bulletin, 130, 793 - 812.

Brown, R. J., \& Oakley, D. A. (2004). An integrative cognitive model of hypnosis and high hypnotisability. In M. Heap, R. J. Brown, \& D. A. Oakley (Eds.), The highly hypnotizable person: Theoretical, experimental and clinical issues (pp. 152-186). London: Brunner-Routledge.

Butler, L. D., Duran, E. E., Jasiukaitis, P., Koopman, C., \& Spiegel, D. (1996). Hypnotizability and traumatic experience: A diathesis-stress model of dissociative symptomatology. American Journal of Psychiatry, 153, 42-63.

Cardeña, E. (1994). The domain of dissociation. In S. J. Lynn, \& J. W. Rhue (Eds.), Dissociation: Clinical and Theoretical Perspectives (pp. 15-31). New York, NY, USA: The Guilford Press.

Cassano, G. B., Petracca, A., Perugi, G., Toni, C., Tundo, A., \& Roth, M. (1989). Derealization and panic attacks: Evaluation on 150 patients with panic disorder/agoraphobia. Comprehensive Psychiatry, 30, 5-12.

Conway, M. A. (2005). Memory and the self. Journal of Memory and Language, 53, 594-628.

Conway, M. A. \& Pleydell-Pearce, C. W. (2000). The construction of autobiographical memories in the self-memory system. Psychological Review, 107, 261-288.

Dell, P. F. (in press). The multidimensional inventory of dissociation (MID): A comprehensive measure of pathological dissociation. Journal of Trauma \& Dissociation.

Frankel, F. H. (1994). Dissociation in hysteria and hypnosis: A concept aggrandized. In S. J. Lynn \& J. W. Rhue (Eds.), Dissociation: Clinical and Theoretical Perspectives (pp. 80-93). New York, NY, USA: The Guilford Press.

Hilgard, E. R. (1977). Divided consciousness: Multiple controls in human thought and actions. New York: Wiley.

Holmes, E., Brown, R. J., Mansell, W., Fearon, R. P, Hunter, E., Frasquilho, F. \& Oakley, D. A. (2005). Are there two qualitatively distinct forms of dissociation? A review and some clinical implications. Clinical Psychology Review, 25, 1-23.

Hunter, E. C. M., Phillips, M. L., Chalder, T., Sierra, M., \& David, A. S. (2003). Depersonalization disorder: A cognitive behavioural conceptualisation. Behaviour Research and Therapy, 41, 1451-1467.

Hunter, E. C. M, Baker, D., Phillips, M. L., Sierra, M. \& David, A. S. (2005). Cognitivebehaviour therapy for depersonalisation disorder: An open study. Behaviour Research and Therapy, 43, 1121-1130.

Hunter, E. C. M., Sierra, M., \& David, A. S. (2004). The epidemiology of depersonalisation and derealisation: A systematic review. Social Psychiatry and Psychiatric Epidemiology, 39, 9-18.

Jahanshahi, M. \& Frith, C. D. (1998). Willed action and its impairments. Cognitive Neuropsychology, 15, 483-533.

Johnson, M. K. \& Raye, C. L. (1981). Reality monitoring. Psychological Review, 88, 67-85.

Kihlstrom, J. F. (1992). Dissociative and conversion disorders. In D. J. Stein, \& J. E. Young (Eds.), Cognitive science and clinical disorders (pp. 247-270). San Diego, CA7 Academic Press. 
Kihlstrom, J. F. (1994). One hundred years of hysteria. In S. J. Lynn, \& J. W. Rhue (Eds.), Dissociation: Clinical and theoretical perspectives (pp. 365-394). New York7 Guilford Press.

Kirsch, I. \& Lynn, S. J. (1997). Hypnotic involuntariness and the automaticity of everyday life. American Journal of Clinical Hypnosis, 40, 329-348.

Kosslyn, S. M. (1996). Image and brain. Cambridge, MA: MIT Press.

Kuyk, J., Spinhoven, P., \& Van Dyck, R. (1999). Hypnotic recall: A positive criterion in the differential diagnosis between epileptic and pseudoepileptic seizures. Epilepsia, 40, 485-491.

Lambert, M. V., Sierra, M., Phillips, M. L., David, A. S. (2002). The spectrum of organic depersonalization: A review plus four new cases. Journal of Neuropsychiatry \& Clinical Neurosciences, 14, 141-154 .

Logan, G. D. (1988). Toward an instance theory of automatization. Psychological Review, 95, 492-527.

Marcel, A. J. (1983). Conscious and unconscious perception: An approach to the relations between phenomenal experience and perceptual processes. Cognitive Psychology, 15, 238-300.

Marshall, R. D., Schneier, F. R., Lin, S., Simpson, H. B., Vermes, D., \& Leibowitz, M. (2000). Childhood trauma and dissociative symptoms in panic disorder. American Journal of Psychiatry, 157, 451-453.

Nijenhuis, E. R. S., Spinhoven, P., Van Dyck, R., Van der Hart, O., \& Vanderlinden, J. (1996). The development and the psychometric characteristics of the Somatoform Dissociation Questionnaire (SDQ-20). Journal of Nervous and Mental Disease, 184, 688-694.

Nijenhuis, E. R. S.

Nijenhuis, E. R. S. (2004). Somatoform dissociation. New York: Norton.

Nijenhuis, E.R.S., Van der Hart, O. \& Steele, K. (2004). Trauma-related structural dissociation of the personality. Trauma Information Pages website, January 2004: http://www.traumapages.com/nijenhuis-2004.htm

Norman, D. A., \& Shallice, T. (1986). Attention to action: Willed and automatic control of behavior. In R. J. Davidson, G. E. Schwartz, \& D. Shapiro (Eds.), Consciousness and self-regulation: Vol. 4. Advances in research and theory (pp. 1-18). New York: Plenum Press.

Noyes, R., \& Kletti, R. (1977). Depersonalization in response to life-threatening danger. Comprehensive Psychiatry, 8, 375-384.

Oakley, D. A. (1999a). Hypnosis and conversion hysteria: A unifying model. Cognitive Neuropsychiatry, 4, 243-265.

Oakley, D. A. (1999b). Hypnosis and consciousness: A structural model. Contemporary Hypnosis, 16, 215-223.

Phillips, M. L., Medford, N., Senior, C., Bullmore, E. T., Brammer, M. J., Andrew, C., et al. (2001). Depersonalization disorder: thinking without feeling. Psychiatry Research. Neuroimaging, 108, $145-160$.

Putnam, F. W. (1997). Dissociation in children and adolescents: A developmental perspective. New York: The Guilford Press.

Saxe, G. N., Van der Kolk, B. A., Berkowitz, R., Chinman, G. et al (1993). Dissociative disorders in psychiatric inpatients. American Journal of Psychiatry, 150, 1037-1042.

Sierra, M., \& Berrios, G. E. (1998). Depersonalization: Neurobiological perspectives. Biological Psychiatry, 44, 898-908.

Sierra, M. \& Berrios, G. E. (2001). The phenomenological stability of depersonalization: Comparing the old with the new. Journal of Nervous and Mental Disease, 189, 629-636.

Sierra, M., Senior, C., Dalton, J., McDonough, M., Bond, A., Phillips, M. L., et al. (2002). Autonomic response in depersonalization disorder. Archives of General Psychiatry, 59, 833-838.

Simeon, D., Gross, S., Guralnik, O., Stein, D. J., Schmeidler, J., \& Hollander, E. (1997). Feeling unreal: 30 cases of DSM-III-R Depersonalization Disorder. American Journal of Psychiatry, 154, 1107-1113.

Sloman, S. A. (1996). The empirical case for two systems of reasoning. Psychological Bulletin, 119, 3-22.

Steinberg, M. (1994). Structured clinical interview for DSM-IV dissociative disorders (SCIDD), revised. Washington, DC: American Psychiatric Press.

Sterlini, G. L., \& Bryant, R. A. (2002). Hyperarousal and dissociation: A study of novice skydivers. Behaviour Research and Therapy, 40, 431-437.

Van der Hart, O. \& Dorahy, M. J. (in press). Dissociation: History of a concept. In P. F. Dell \& J. O'Neill (Eds.), Dissociation and the dissociative disorders: DSM-V and beyond. Chicago: International Society for the Study of Dissociation. 
Van der Hart, O., Nijenhuis, E., Steele, K. \& Brown, D. (2004). Trauma-related dissociation: Conceptual clarity lost and found. Australian and New Zealand Journal of Psychiatry, 38, 906-914.

Van der Kolk, B. A., \& Fisler, R. (1995). Dissociation and the fragmentary nature of traumatic memories: Overview and exploratory study. Journal of Traumatic Stress, 8, 505-525.

van Ijzendoorn, M.H. \& Schuengel, C. (1996). The measurement of dissociation in normal and clinical populations: Meta-analytic validation of the Dissociative Experiences Scale (DES). Clinical Psychology Review, 16, 365-382.

Velmans, M. (2000). Understanding consciousness. London: Routledge.

World Health Organization. (1992). The ICD-10 classification of mental and behavioural disorders: Clinical descriptions and diagnostic guidelines. Geneva, Switzerland: Author. 


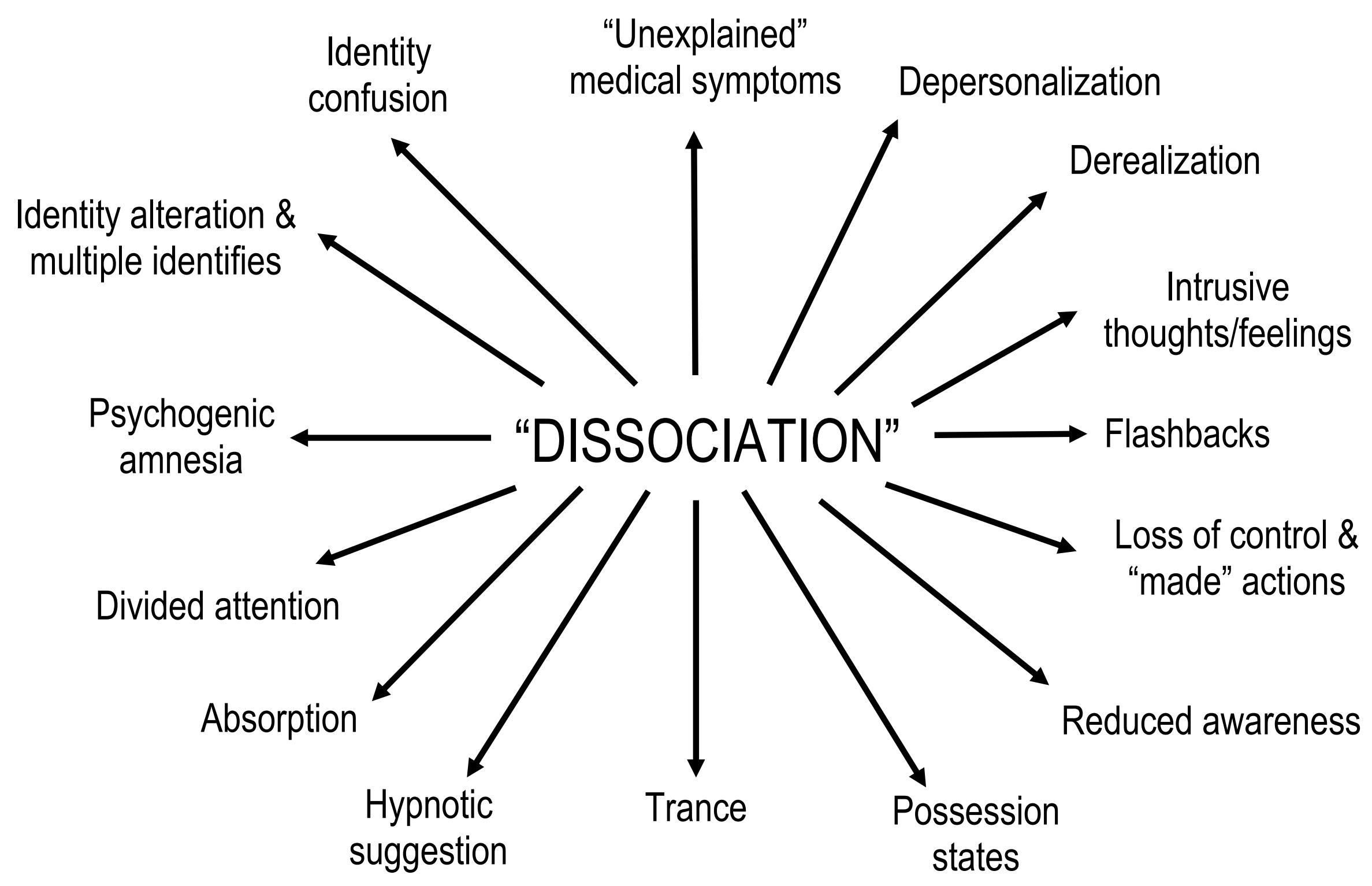


Figure 2: $\quad$ Hypothetical dissociative continuum (not to scale). N.B. The inclusion of both states and disorders on a single dimensional scale is deliberate in order to illustrate the assumption underlying the unitary model, viz that different dissociative states and conditions can be regarded as involving different "amounts" of dissociation
Absorbed
Hypnotic
Dissociative
phenomena
amnesia
Dissociative identity
states
disorder

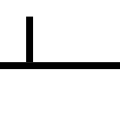

1
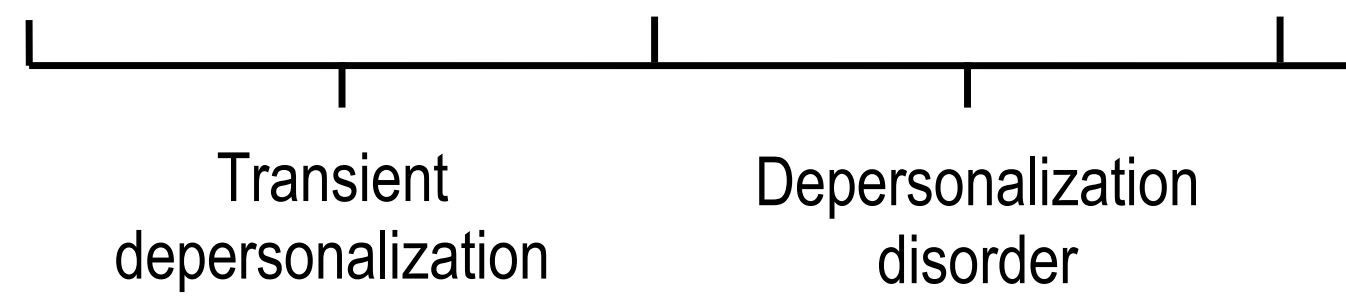

Depersonalization

disorder

Somatization

disorder

Increasing "amount" of dissociation 
Table 1: Detachment and compartmentalization phenomena

\begin{tabular}{ll}
\hline Detachment phenomena & Compartmentalization phenomena \\
\hline Emotional numbing & Unexplained neurological symptoms \\
Depersonalization & Hypnotic phenomena \\
Derealization & "Made" actions \\
Out-of-body experiences & Multiple identities \\
Amnesia due to encoding deficit & Amnesia due to retrieval deficit \\
& Identity confusion* \\
\hline
\end{tabular}

\footnotetext{
* Identity confusion is a non-specific symptom that can be associated with either detachment or compartmentalization
} 
Figure 3: $\quad$ Structures and processes involved in the generation of consciousness and control of cognition and action

EARLY PERCEPTUAL PROCESSES

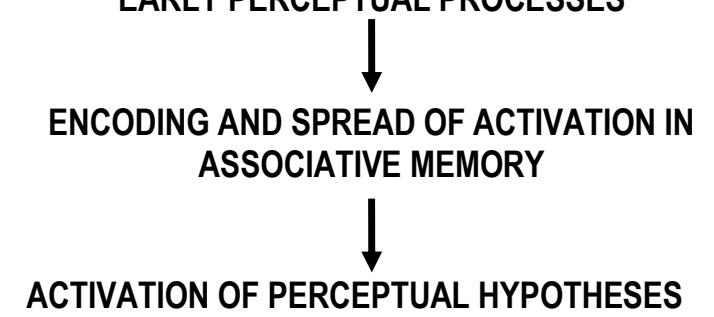

Synthesis of hypotheses with sense data

PRIMARY ATTENTIONAL SYSTEM (PAS)

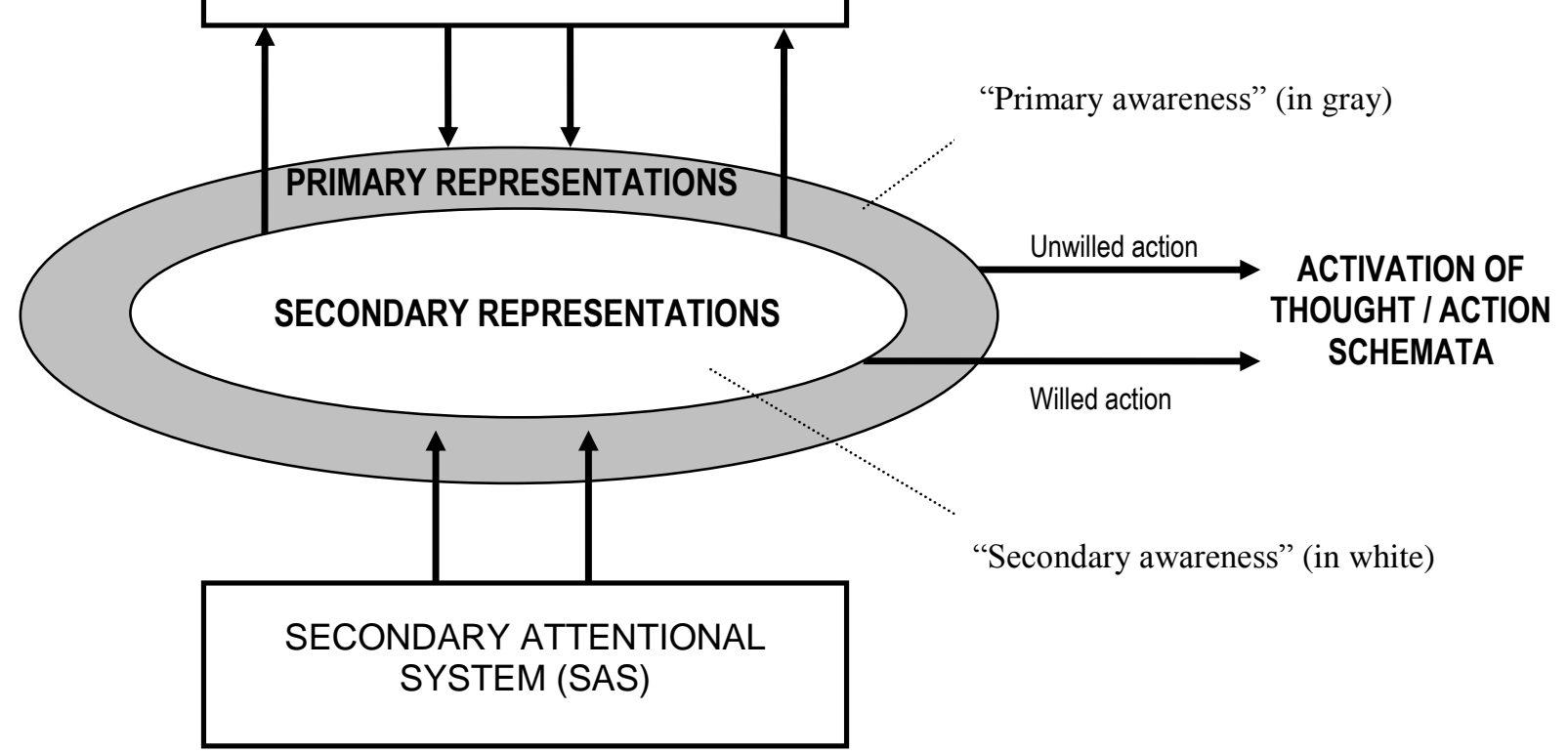

